\title{
Anisotropic Materials Appearance Analysis using Ellipsoidal Mirror
}

\author{
Jiří Filip ${ }^{a}$ and Radomír Vávra ${ }^{a, b}$ \\ ${ }^{a}$ Institute of Information Theory and Automation of the Czech Academy of Sciences, \\ Pod Vodárenskou věží 4, 182 08, Praha 8, Czech Republic; \\ ${ }^{b}$ Faculty of Information Technology, Czech Technical University in Prague, \\ Thákurova 9, 16000 Praha 6, Czech Republic
}

\begin{abstract}
Many real-world materials exhibit significant changes in appearance when rotated along a surface normal. The presence of this behavior is often referred to as visual anisotropy. Anisotropic appearance of spatially homogeneous materials is commonly characterized by a four-dimensional BRDF. Unfortunately, due to simplicity most past research has been devoted to three dimensional isotropic BRDFs. In this paper, we introduce an innovative, fast, and inexpensive image-based approach to detect the extent of anisotropy, its main axes and width of corresponding anisotropic highlights. The method does not rely on any moving parts and uses only an off-the-shelf ellipsoidal reflector with a compact camera. We analyze our findings with a material microgeometry scan, and present how results correspond to the microstructure of individual threads in a particular fabric. We show that knowledge of a material's anisotropic behavior can be effectively used in order to design a material-dependent sampling pattern so as the material's BRDF could be measured much more precisely in the same amount of time using a common gonioreflectometer.
\end{abstract}

Keywords: BRDF, anisotropy, detection, setup, mirror, ellipsoidal

\section{INTRODUCTION}

Anisotropic materials are, due to their atypical rich appearance, often used in achieving an eye-catching look of many man-made products as shown in Fig. 1. Fabrics for fancy apparel are probably the most typical example. Anisotropic finishing of plastics or metals is often used to create resonant appearance in personal items or design electronics. Genuine wooden materials are also very popular due to their specific anisotropic behavior.
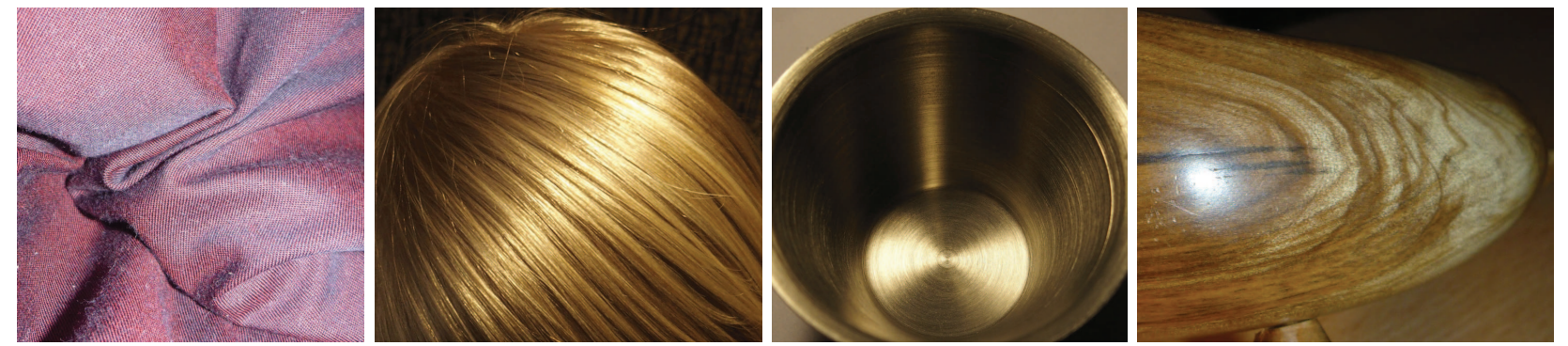

Figure 1. Real-wold examples of anisotropic appearance of fabric, hair, brushed metal, and polished wood caused by the orientation of cylindric threads/hair fibers, parallel grooves in metal (both causing highlights perpendicular to their direction), and wooden fibers (exhibiting both specular (left) and anisotropic (right) highlights), respectively.

In general, anisotropy is the property of being directionally dependent, as opposed to isotropy, which implies identical properties in all directions. It is most often observed in chemistry in the evaluation of optical properties in different crystallographic directions or in physics for the interpretation of the speed of light in materials in

Further author information: (Send correspondence to J.F.)

J.F.: E-mail: filipj@utia.cas.cz, Telephone: +42026605 2365

R.V.: E-mail: vavra@utia.cas.cz, Telephone: +42026605 2224 
various direction. In image processing, isotropy and anisotropy are used in the analysis of a directional material's appearance as well as capabilities of related data processing methods. When a material's reflectance is constant for fixed view and illumination irrespective to the rotation of the material around its normal, the material is considered isotropic. In contrast, materials whose reflectance is not constant are considered anisotropic. This also corresponds to measurement and modeling methods that can be categorized into two groups depending on their ability to measure or model anisotropic behavior.

Digital material appearance offers a number of representations. They range from well established reflectance representations such, e.g., BRDF (Bidirectional Reflectance Distribution Function) ${ }^{1}$ to more complex texture and light scattering-based, e.g., BTF (Bidirectional Texture Function), ${ }^{2}$ BSSRDF (Bidirectional Surface Scattering Reflectance Distribution Function). ${ }^{1}$ While the latter group captures material appearance in all its complexity including shadowing, masking, inter-reflections, subsurface scattering, the former is based on simplified assumptions of opaque and flat materials which omit any textural information. However, many appearance measurement and modeling approaches go even further and reduce their processing costs by sacrificing material anisotropy.

In this paper we study global material reflectance as represented by the BRDF introduced by Nicodemus et al. ${ }^{1}$ describing the ratio of energy reflected by the material for certain combination of incoming $\omega_{i}=\left[\theta_{i}, \varphi_{i}\right]$ and outgoing $\omega_{v}=\left[\theta_{v}, \varphi_{v}\right]$ directions. Incoming and outgoing directions are specified by $\theta$ elevation and $\varphi$ azimuthal angles. When we assume separate processing of color channels, the anisotropic BRDF is a four-dimensional function $B\left(\theta_{i}, \varphi_{i}, \theta_{v}, \varphi_{v}\right)$, while the isotropic BRDF is three-dimensional only, i.e., $B\left(\theta_{i}, \theta_{v}, \varphi_{i}-\varphi_{v}\right)$. Fig. 2 compares BRDFs of isotropic (a) and anisotropic (b) materials. Row/columns of the BRDF images correspond to illumination/viewing directions circling hemisphere above the material from top to bottom (see ${ }^{3}$ for detailed BRDF image description). While for isotropic material the locations of specular highlights can be predicted at configurations where light shines opposite to camera (see diagonal lines in BRDF in Fig. 2-(a)), a number and locations of anisotropic highlights for anisotropic materials are unknown depending entirely on material micro-structure and its orientation during BRDF measurement process.

(a)
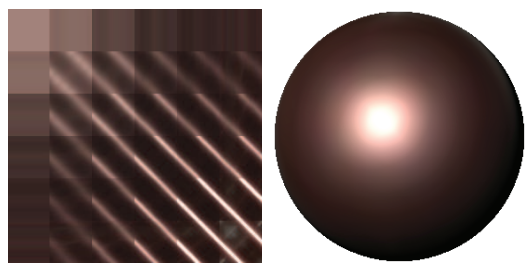

(b)
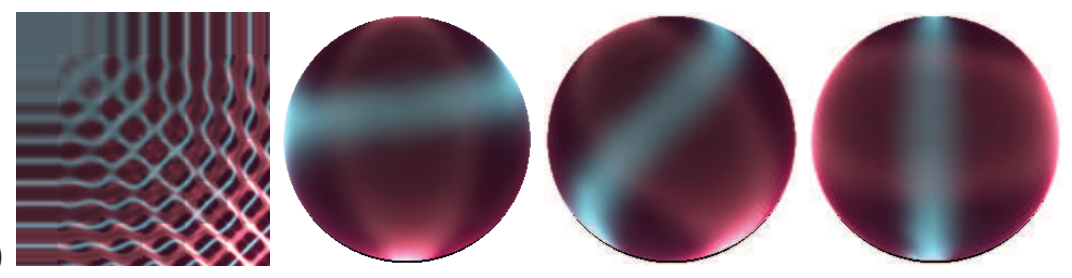

(a) isotropic metallic paint, (b) anisotropic fabric with two anisotropic highlights. Renderings show the dependency of anisotropic highlight locations on material orientation as defined by an object's tangential space, while sphere's orientation remains fixed.

Why is the capturing of visual anisotropy difficult? It is primarily due to the fact that the capturing of another dimension enormously expands measurement state space when compared to the isotropic acquisition of material. In the case of BRDF one has to capture four dimensions instead of three. Therefore, it is time demanding to sample this space uniformly while still maintaining visual quality comparable to isotropic measurements.

In this paper we study materials' visual anisotropy and introduce its inexpensive and fast detection technique based only on an ellipsoidal mirror and compact camera. Our technique allows us to determine strength of anisotropy, main anisotropy axes, and the width of anisotropic highlights.

The paper first overviews anisotropy related research in Section 2. Section 3 introduces a novel method of anisotropic behavior detection, the test datasets, and achieved results. Moreover, it discusses methods advantages and limitations. Section 4 illustrates application of the acquired information to sampling of anisotropic BRDFs. Section 5 concludes the paper.

\section{PRIOR WORK}

Most BRDF related research is, due to its simplicity, limited to isotropic materials only. Although, first models accounting for visual anisotropy appeared in the early nineties, ${ }^{4}$ the analysis of anisotropic behavior only received 
attention much later. An initial analysis of material anisotropy studying optical properties of velvet was done by $\mathrm{Lu}$ et al. ${ }^{5}$ In their follow-up work, ${ }^{6}$ the authors predicted locations of anisotropic reflections on cylinders, cones and spheres made of materials containing tangential hairs or grooves. Only recently has the notable CookTorrance micro facet-based model $^{7}$ (derived from ${ }^{8}$ ) been extended to the modeling of anisotropic reflections. ${ }^{9}$ The newest work by Raymond et al. ${ }^{10}$ exploits knowledge of anisotropic reflections behavior for the user manipulation of anisotropic highlights location employing the modification of tangential fields on arbitrary geometry.

In similarity to BRDF research, anisotropic BRDF measurements are relatively rare. Initially such measurement were introduced in the nineties. ${ }^{4,5}$ The first four public anisotropic measurements were published by Ngan et al. ${ }^{11}$ Their accuracy, however, was limited due to a lower reliability of samples acquired at oblique viewing angles, and a more accurate dense BRDF measurements of three fabric materials were introduced by Filip et al. ${ }^{12}$ Subsequently, the same authors introduced the first database of 150 anisotropic BRDFs. ${ }^{3}$

As our anisotropy detection setup is mirror-based, we briefly observe previous work in the field of anisotropic BRDF measurement. It can be divided into several groups depending on which type of mirror used. They used either kaleidoscopically arranged flat mirrors, ${ }^{13}$ hemispherical mirrors, ${ }^{4}$ off-axis parabolic mirrors, ${ }^{14}$ ellipsoidal mirrors, ${ }^{15,16}$ or a combination of concave parabolic and a custom-built mirrors. ${ }^{17}$ Another approach called hemispherical confocal imaging uses an assortment of flat mirrors distributed over an ellipsoid. ${ }^{18}$ It can be viewed as a set of virtual cameras and projectors positioned uniformly over the hemisphere. All these setups place the measured material into the focal point of the mirror, share the optical and illumination axis using coaxial pair of camera and projector, and thus allow the recording of multiple illumination or view directions in a single image. The main advantage of such an arrangement is the elimination of any mechanical component in the measurement setup. Principal disadvantages are: often a limited range of recorded elevation angles, variable reflectance attenuation across the elevations, or a low dynamic range of the measurements when projector is used as illumination source. Most setups place the material into a mirror focal point in such a way that the material is aligned with the axis of the mirror. ${ }^{14,15,18}$ This is not ideal for fast anisotropy detection as it requires sample extraction and positioning inside the mirror. More applicable are setups aligning the mirror axis with the surface normal; ${ }^{4,19}$ therefore, this approach is adopted in our work.

Our anisotropy detection method is related to the aggregated BRDF measurements presented in; ${ }^{14}$ although we do not use an off-axis parabolic mirror and the method requires merely an inexpensive camera and off-the-shelf ellipsoidal reflector placed directly on the measured sample surface.

Main advantages of the proposed anisotropy detection setup are its low acquisition cost and extremely fast data measurement and analysis of main anisotropy axes and width of highlights.

\section{THE PROPOSED ANISOTROPY ANALYSIS APPROACH}

The most straightforward way of detecting anisotropy presence/type is to mutually fix light and sensor positions while rotating the measured sample beneath them, while recording outgoing reflectance by the sensor. However, it is possible that this can be done more quickly and without any mechanical movement. Our anisotropy detection technique originates out of mirror-based setups; however, it is considerably simplified. It consist of an ellipsoidal reflector, with an opening at the narrowest part Fig. 3-bottom-left. It is then placed on the measured material. This reflector is photographed by a compact camera having an optical axis approximately aligned with the reflector axis that also represents the normal of the measured surface.

In contrast to other setups, we do not attempt to record the material's BRDF, but only its anisotropic behavior. Therefore, we do not sweep the mirror by a controlled ray of light from the projector, but use instead the flash from our camera. We use, similarly to, ${ }^{14}$ aggregated illumination which is, however, achieved by a single flash shot. We do not use any special gantry and the image is taken from a distance $1.8 \mathrm{~m}$. This is done in order to capture the reflector (in diameter $11 \mathrm{~cm}$ ) approximately symmetrically, i.e., with the opening in the center of the reflector. Note that our configuration, as shown in inset of Fig. 3, is a reasonable approximation of the coaxial setup as the distance between lens and flash is $7.5 \mathrm{~cm}$.

Such an omni-directional illumination lights the measured material from many azimuthal angles. As the material sample is not positioned in the mirror's focal point, illumination elevations angles vary across the opening (see Fig. 3-right). This allows for the capturing of the azimuthal direction of the anisotropic reflection. 
In the image of material at the opening ( $26 \mathrm{~mm}$ wide) taken by the camera is each location illuminated from two elevations at azimuths 180 degrees apart. Contributions of these two elevations are summed in the captured photo. Mean illumination elevation angle across the entire material plane visible in the opening is 50 degrees. When an isotropic material is analyzed, the captured image contains a circularly shaped peak in the center. This is caused by the accumulation of reflected rays at that minute location. However, for an anisotropic material, we additionally observe a couple of triangle shaped highlighted areas running symmetrically from the center to the edges of the opening as shown in the first row of Fig. 5. Their azimuthal orientation coincides with the direction(s) of the main anisotropy axis(axes), while their width corresponds to width of the anisotropic highlight for the illumination elevation 50 degrees. Thus, when an unknown material is observed we can easily determine the presence of anisotropy, its shape, and, azimuthal direction.
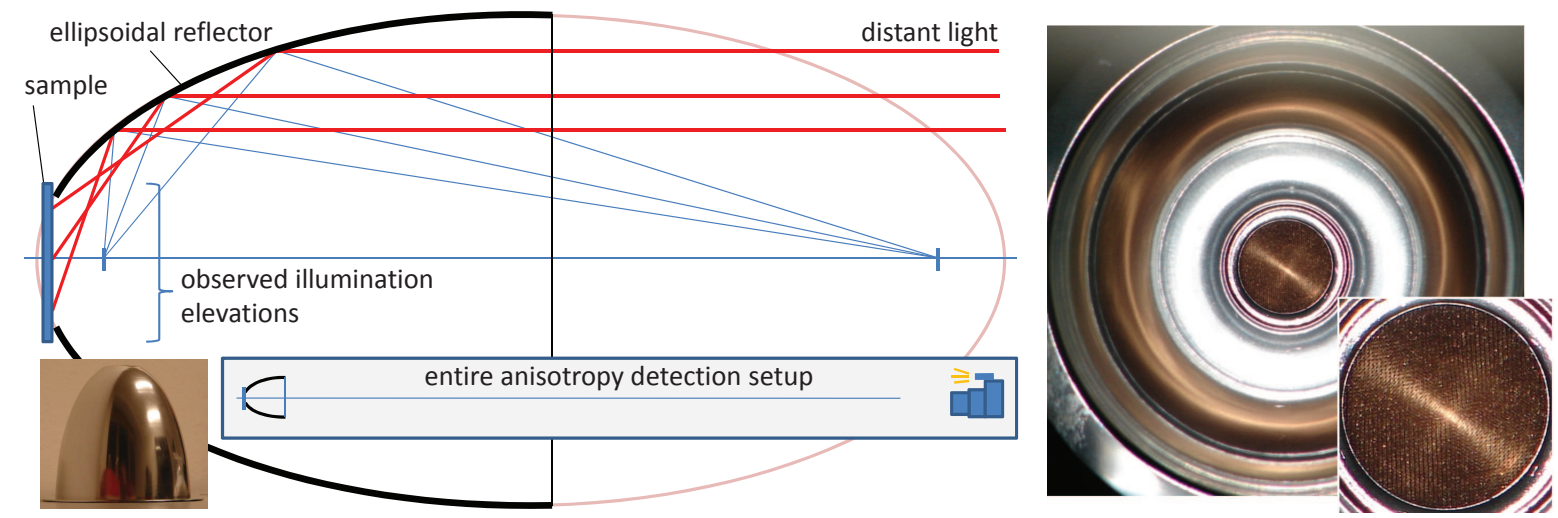

Figure 3. Scheme of anisotropy detection using ellipsoidal reflector (left) and image captured by a camera (right).

\subsection{Tested Materials}

To test our approach, we used eight complex anisotropic fabric materials and compared their microstructure scans with the detected anisotropy direction recorded by our method. For our test samples we used eight fabric materials from the UTIA BRDF database*. ${ }^{3}$ All of them consist of two (same or different) interwoven threads and all materials exhibit strong specific anisotropic behavior as shown in their rendering in the first row of Fig. 4. The second row shows their BRDFs. The third row of the figure shows the detailed zoom into the materials' micro-structure and reveals the threads and weaving pattern that influence the materials' anisotropic behavior. Primary and secondary (if applicable) anisotropy axes are shown by green and blue arrows respectively.

The main anisotropy axis of materials consisting of two identical threads (fabric111, fabric134) is determined by the weaving pattern. The main axis is orthogonal to thread occupying the upper layer of the material. These materials have also distinctive secondary anisotropic axis (shown as a vertical highlight in the renderings in Fig. 4). Other materials consisting of nylon fibers exhibiting the highest reflectivity orthogonal to their upper threads (fabric094) or nylon fibers (fabric135, fabric136) direction. Finally, the materials consisting of similar threads of different colors (fabric002, fabric106, fabric112) exhibit an intricate color shifting appearance depending on the actual illumination and viewing direction of the weaving structure. In fact, these materials have two anisotropic highlights; although the second one is very wide and difficult to detect at elevations below $60^{\circ}$.

\subsection{Results}

The results obtained for tested materials are shown in the first row of Fig. 5. The first row shows the opening in the mirror as observed by the camera. It is apparent this simple approach is capable of detecting a number of anisotropic highlights, their colors, direction, and width. Indeed, this image provides very descriptive information about the material anisotropic behavior without the necessity of a lengthy measurement. Note the striking resemblance with renderings of the respective materials' BRDFs in Fig. 4. The second row shows these images

${ }^{*}$ http://btf.utia.cas.cz 


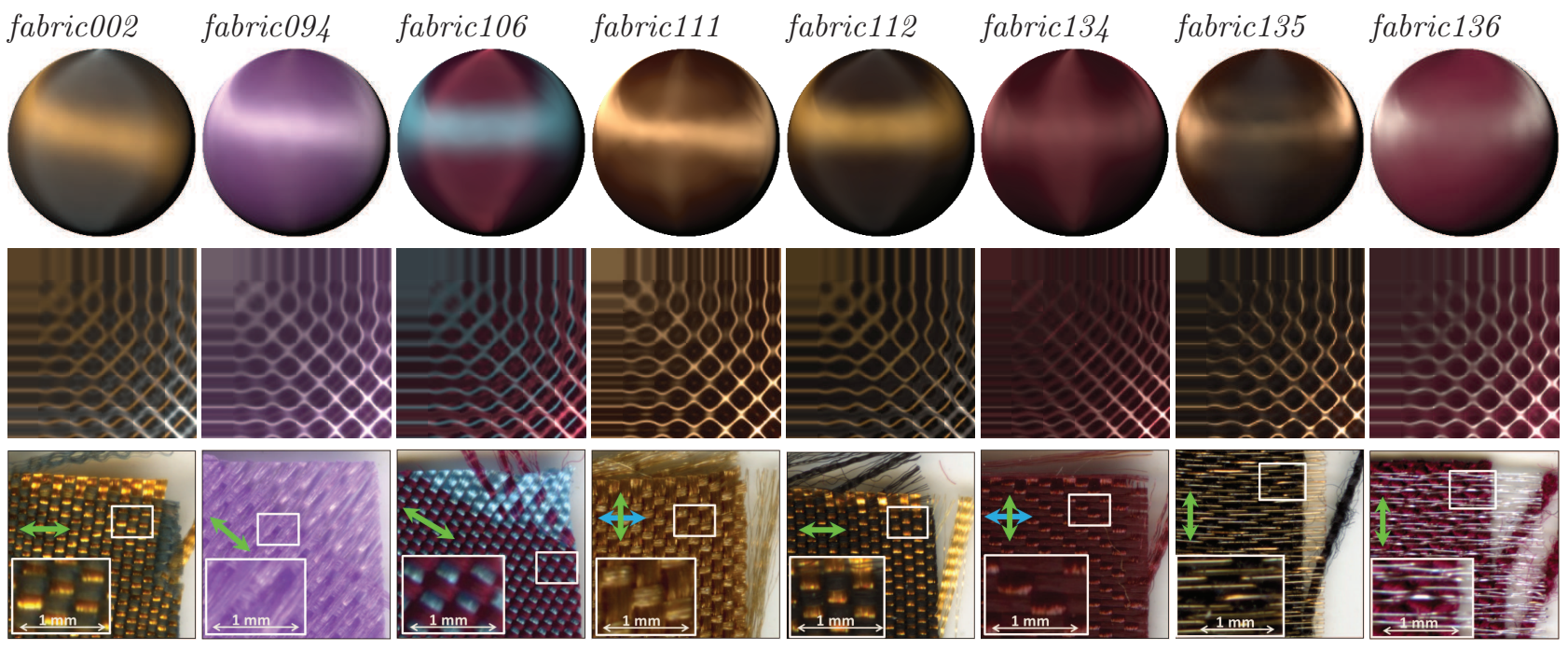

Figure 4. The materials rendered on sphere for point light illumination (the first row), their BRDFs (the second row). Detailed zoom into micro-structure of the tested materials with marked primary (green) and secondary (blue) anisotropy axes (the third row).

after filtering and smoothing by median and box filters. Additionally, these images illustrate the automatically detected axes of anisotropy (main as green, secondary as blue), defined by the azimuthal angle $\varphi_{h}$, as well as the estimated width of anisotropic highlight. Each such image can be viewed as a polar plot which can be transformed to a Cartesian plot as shown in the third row. These Cartesian plots show reflectance from the edge to center of the opening based on the azimuthal illumination angle. In the Cartesian plots, the increase of intensity towards

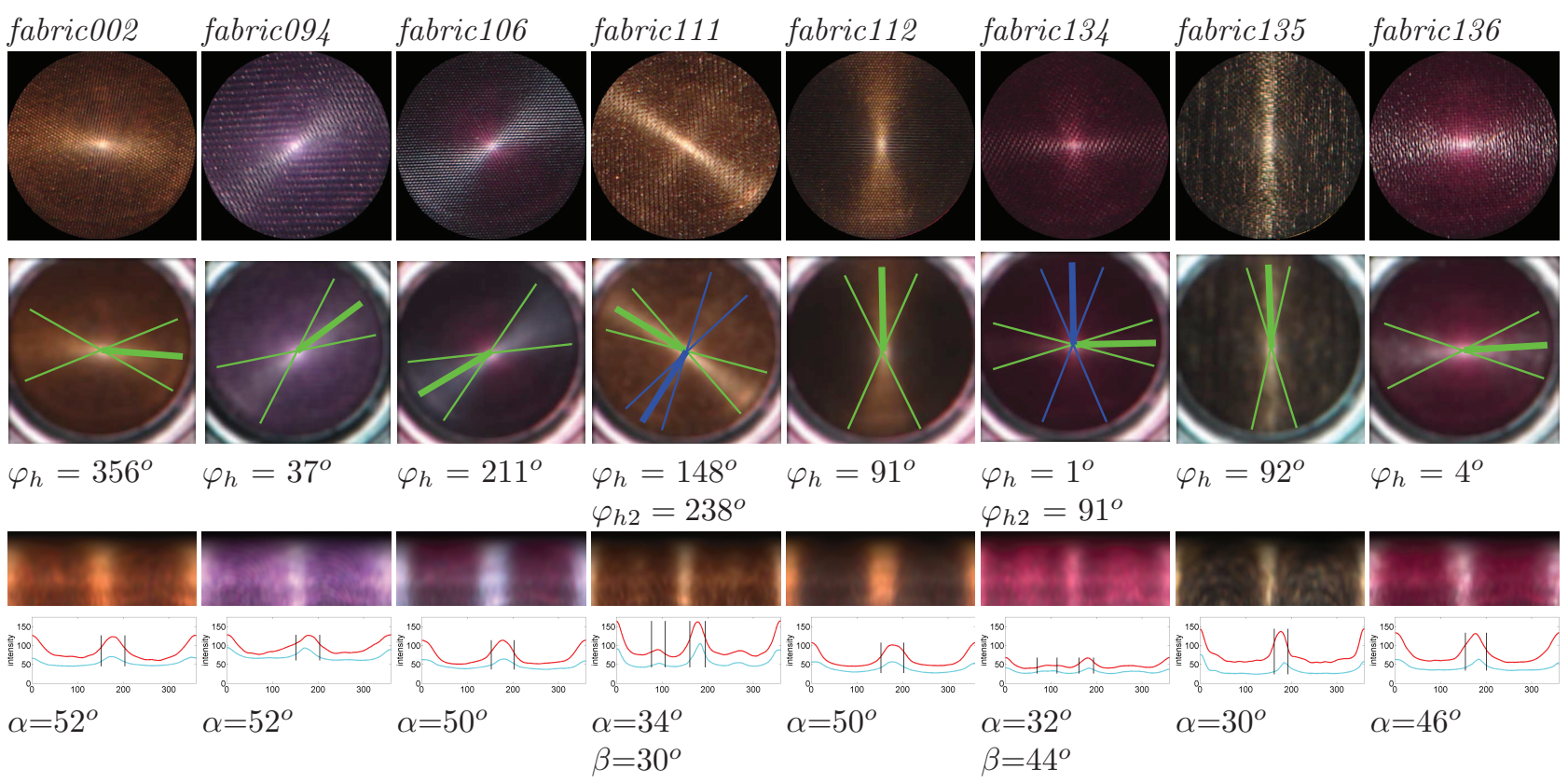

Figure 5. Anisotropy presence/type detection using the proposed method: the first row - captured image of the reflector opening, the second row - its smoothed version with detected anisotropic highlights (green - primary anisotropy axis, blue - secondary anisotropy axis), the third row - radius $/ \varphi_{h}$ image with clear vertical highlight (with compensated isotropic behavior), the fourth row - 1D plot of anisotropic highlight profile (red line) with estimated width of anisotropic highlights $\alpha, \beta$. The reference BRDF values are shown as cyan lines. 
the images center is compensated for using an image of a Lambertian-like black paper sample and all images are aligned according to their main anisotropic highlight. Note that this is not necessary when only the lower part of the images is used. In these images we can observe a different width and shape of anisotropic highlights. One can average images in the third row along a vertical axis and obtain 1D profiles of anisotropic highlights as shown in the fourth row. Given the predefined threshold, we can estimate the width of the highlights (red line). In validation of our method, we can observe a very close resemblance of the averaged values (red line) to the know reference BRDF values corresponding to $\theta_{v}=0$ and $\theta_{i}=50$ (cyan line).

\subsection{Advantages}

The main advantage of the proposed technique is fast measurement and processing, which takes $4.4 \mathrm{~s}$ using single core of Intel Core i7. Another advantage is the low setup acquisition cost as only an of-the-shelf ellipsoidal mirror (price $\$ 7$ ) and a basic compact camera with sufficient zoom and resolution are needed. A final notable advantage is the fact that the setup does not require any calibration procedure apart from attaching the reflector to the measured nearly planar surface and taking its picture from a distance $1.5 \mathrm{~m}$ with a forced flash in lower ambient lighting conditions.

\subsection{Limitations}

The proposed anisotropy detection approach is limited to nearly homogeneous and almost planar materials, i.e., without significant spatially varying appearance whose effects like texture or shadows would visually mask anisotropic highlights. Therefore, we have experimented with different smooth fabric materials that avoid this limitation while still providing a wide range of available types of anisotropic appearance. Similarly one can use this method for the analysis of any flat groove- or fibers-based types of anisotropy, e.g., present in wooden or in machined or polished surfaces.

As the mirror detects anisotropy corresponding to elevation angle $50^{\circ}$ it cannot principally detect anisotropic highlights which are not apparent at such an elevation. This is the case in materials fabric002, fabric106, and fabric112, where a wide width of secondary anisotropic highlight serves to make its distinguishing from the background reflectance quite difficult.

\section{APPLICATION TO BRDF SAMPLING}

The results of the proposed anisotropy analysis were applied to improve BRDF sampling. Instead of simplistic uniform sampling, we suggest using obtained information about anisotropic highlight(s) position and their shape to develop a custom-based sampling pattern.

When the azimuth of anisotropy axis $\varphi_{h}$ is estimated using the proposed setup, the locations of anisotropic highlights can be predicted on hairs or grooves, laying in planes orthogonal to the bisector (often denoted as half-way direction $\omega_{h}=\frac{\omega_{i}+\omega_{v}}{2}$ ) of directions of incidence and reflectance. ${ }^{6}$ Therefore, the anisotropic highlight is present at directions of the bisector that are orthogonal to the detected anisotropy axis of the material $u=\left[\sin \varphi_{h}, \cos \varphi_{h}, 0\right] .{ }^{10}$ Such a condition can be expressed using

$$
\omega_{h} \cdot u=0
$$

A visualization of functions $\omega_{h} \cdot u,\left|\omega_{h} \cdot u\right|$ and its thresholded variant $\left|\omega_{h} \cdot u\right|<\epsilon$ are shown in Fig. 6-(b,c,d), respectively.

The equation (1) provides us with a center of anisotropic highlight; however, for its proper sampling, one needs to also sample its surroundings. Its distance from the anisotropic highlight should correspond to the width of the anisotropic highlights $\alpha, \beta$ estimated using our setup as well. As the shape of the function $\omega_{h} \cdot u$ for all measured combinations of incidence and reflectance directions can be described by means of a two-dimensional sinusoidal pattern as shown in Fig. 6-(a,b). Highlights are represented by directions where the function crosses zero and the location of such neighborhood directions can be obtained for those $\omega_{h}$ where

$$
\omega_{h} \cdot u=\sin \frac{\alpha}{2} .
$$




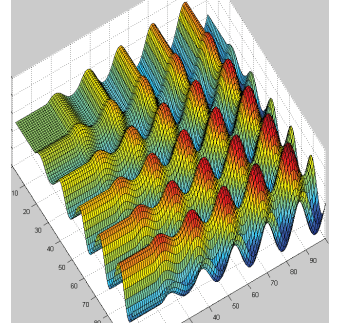

(a) $\omega_{h} \cdot u$

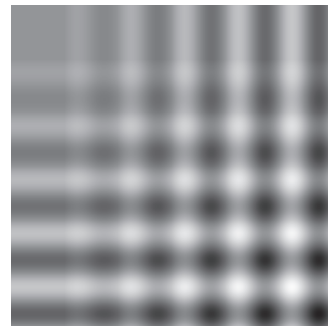

(b) $\omega_{h} \cdot u+1$

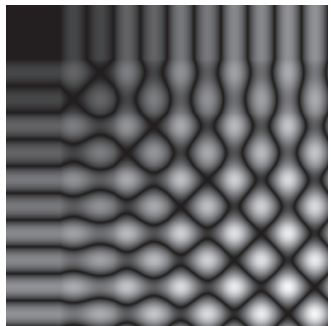

(c) $\left|\omega_{h} \cdot u\right|$

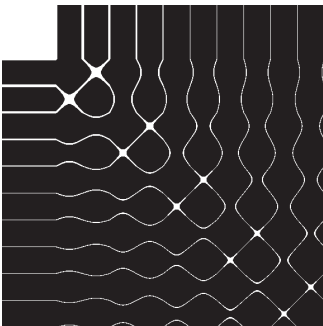

(d) $\left|\omega_{h} \cdot u\right|<0.05$

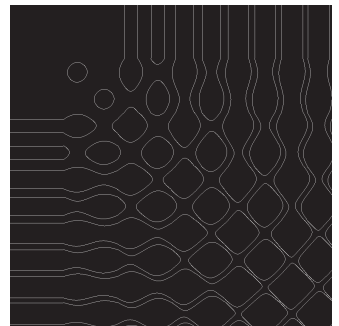

(e) $\left|\omega_{h} \cdot u\right|=\sin \frac{\alpha}{2}$

Figure 6. Predicting of anisotropic highlight location using half-way direction $\omega_{h}$ and the detected direction of anisotropy $u=\left[\sin \varphi_{h}, \cos \varphi_{h}, 0\right]$, where $\varphi_{h}=0^{\circ}$.

Example of such neighboring contours are shown in Fig. 6-(e).

Now the estimated contours of anisotropic highlights with its neighborhoods can be applied in order to improve of sampling of unknown BRDF. For simplicity, we explain our sampling method on BRDF subspace only obtained by fixing illumination and viewing elevations $\theta_{i}=45^{\circ} / \theta_{v}=75^{\circ}$. Fig. 7-a depicts the unknown reference BRDF subspace. Fig. 7-c shows estimated anisotropic highlight with two neighboring contours in BRDF subspace for fixed view and illumination elevation angles. The number of samples over the contours has to be reduced to achieve a reasonable number (e.g., comparable with uniform sampling Fig. 7-b). Therefore, we successively add new samples on the contours in a way so as to maximize their mutual distance as shown in the second image in Fig. 7-c. To also capture reflectance background outside of highlights, we included an additional sample in each subspace at the location most distant from all other highlight samples. Note that for materials exhibiting two anisotropic highlights, is the process of contours estimation done for each highlight separately. However, the sample candidates of the secondary highlight laying at intersections with the main highlight are disregarded. This is due to the fact that the main highlight is always defining the final reflectance at such locations.

(a) reference

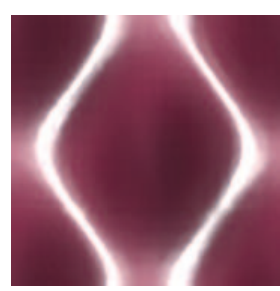

(b) uniform sampling 121 samples

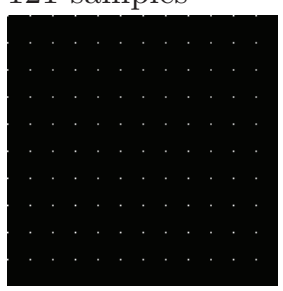
interpolation

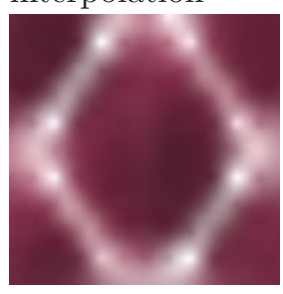

(c) proposed anisotropic sampling

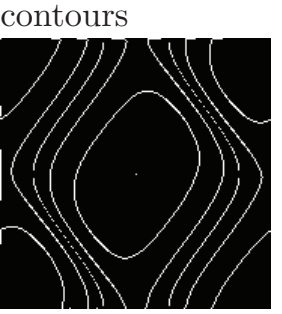

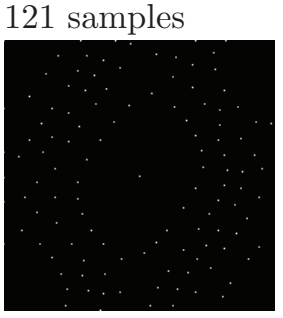

Figure 7. Comparison BRDF subspace (a) reconstruction using uniform sampling (b) and proposed anisotropic sampling (c) using 121 samples. The subspace corresponds to fixed elevation angles $\theta_{i}=45^{\circ} / \theta_{v}=75^{\circ}$.

For our experiments, we use UTIA BRDF Database ${ }^{\dagger}$ benchmark data $^{12}$ that provide dense BRDF measurements with an angular resolution of $2^{\circ}$. This data consists of three BRDFs of fabric materials: fabric112, fabric135, fabric136.

Fig. 7 demonstrates a better performance of the proposed anisotropic sampling approach over uniform sampling of BRDF subspace of fabric136 using the same number of samples. Note that the proposed sampling approach relies on using the detected anisotropy axis direction and highlight width. We applied a four-dimensional radial basis function interpolation to reconstruct BRDF from the samples.

Fig. 8 compares reconstruction performance of entire BRDFs using uniform sampling and the proposed anisotropic sampling, both using 1025 reciprocal samples. Our results, when compared to uniform sampling,

${ }^{\dagger}$ http://btf.utia.cas.cz 
demonstrate a better smoothness of reflectance function and continuity of anisotropic highlights. We also compared the reconstructed and reference BRDFs using computational measures (RMSE,PSNR,VDP2) and found a notable visual improvement, when the detected information about material anisotropy was used for the generation of a proper sampling pattern. A lower performance of the proposed method for fabric135 BRDF is given due to RBF interpolation that, due to a high dynamic range of anisotropic highlights, undershoots the background reflectance near its contours. Therefore, further investigation of other interpolation approaches continues preoccupy our future work endeavors.

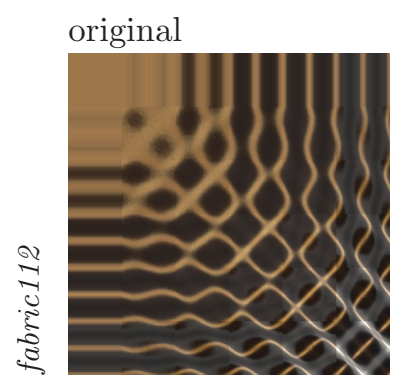

uniform

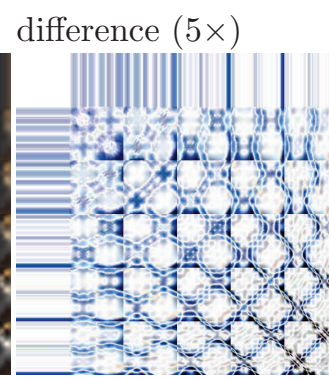

proposed

difference $(5 \times)$

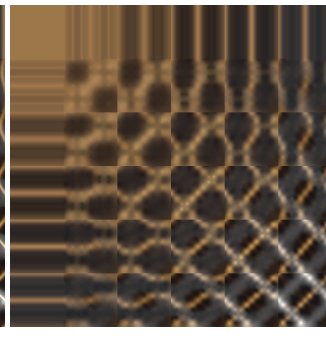

$14.8 / 24.78 / 83.1$
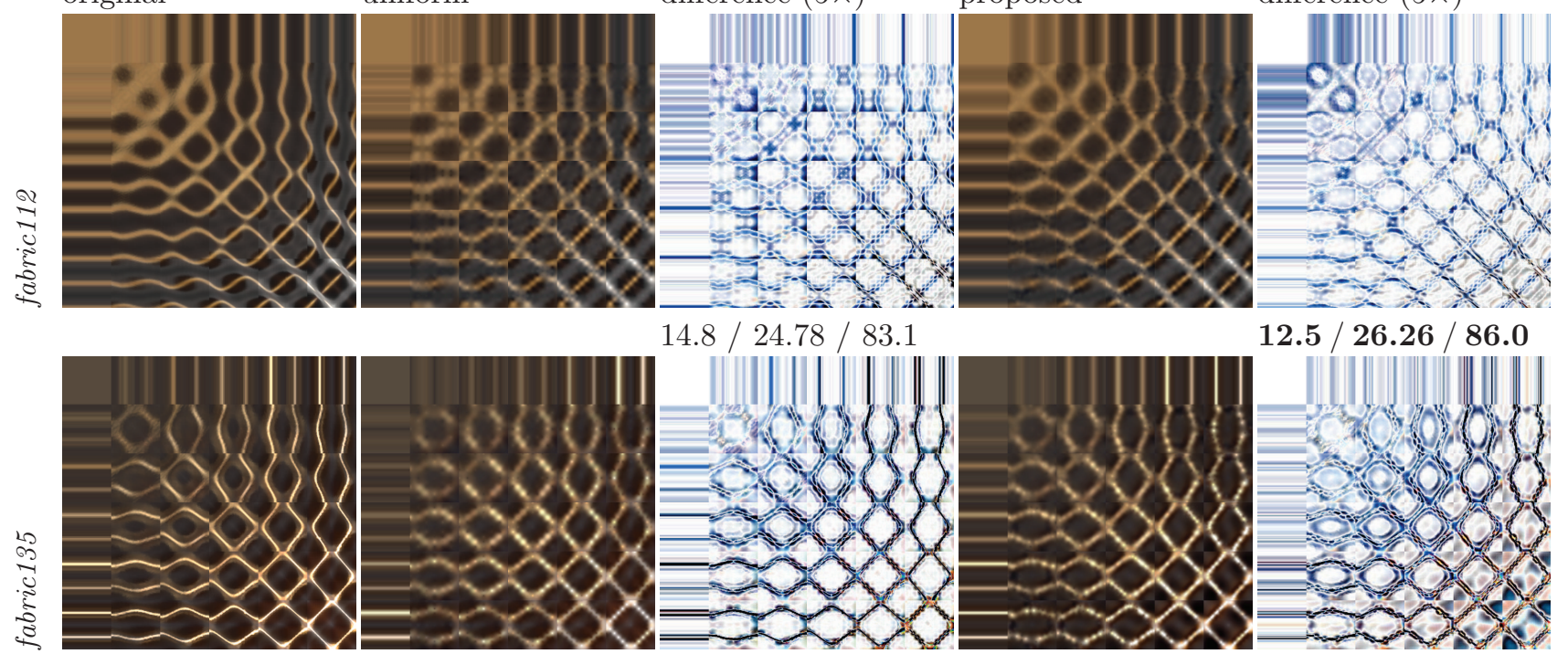

$12.5 / 26.26 / 86.0$
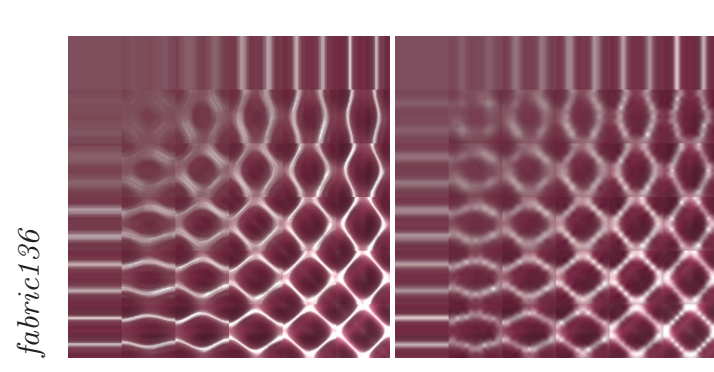

$23.2 / 20.87 / 69.6$
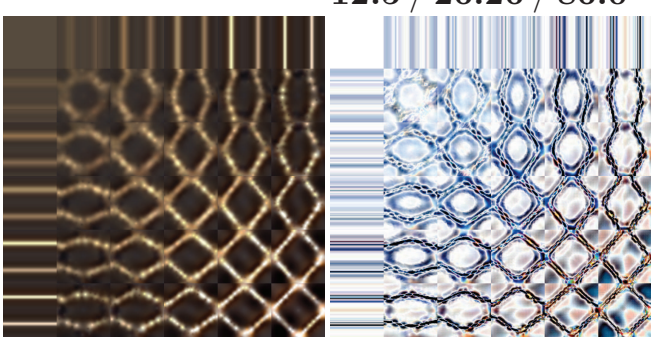

$21.7 / 21.44 / 78.7$

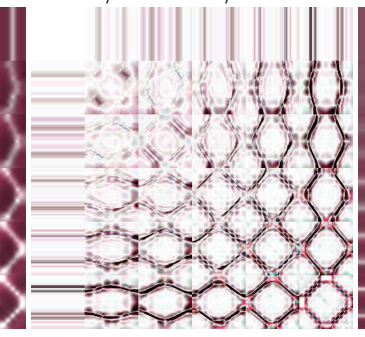

14.5 / $24.97 / 86.8$

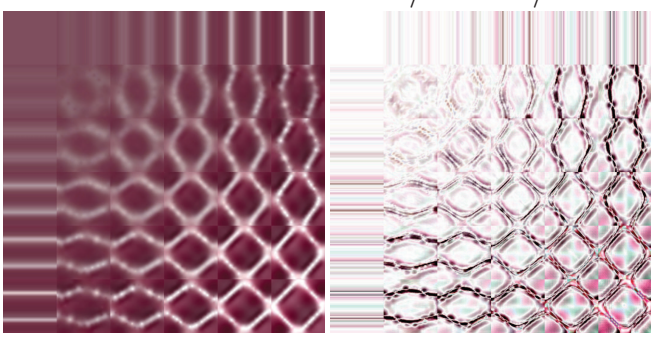

13.4 / $25.62 / 88.3$

Figure 8. Comparison of reference BRDF and its reconstructions using 1025 uniform and 1025 anisotropic reciprocal samples, respectively. Below are shown results of RMSE / PSNR [dB] / VDP2 computational metrics.

Finally, we compared renderings of the same BRDFs in the same scenario comprising four spheres as shown in Fig. 9. Again, the proposed method provides better azimuthal continuity and overall smoothness, which is also supported by the results of the computational measures.

\section{CONCLUSIONS}

We introduced a novel method for convenient and fast detection of materials' anisotropy strength, main anisotropy axes, and corresponding highlights shape. The proposed detection setup consists of an ellipsoidal mirror and compact camera which allows for almost a real-time analysis of material anisotropy without the necessity of extracting a sample from its environment. Furthermore, we show the application of parameters estimated using our method for improving unknown BRDF sampling. We believe that the proposed fast analysis of anisotropic appearance will be beneficial for variety of tasks, ranging from improvement of material appearance acquisition (as shown in this paper) to its automatic classification or image-based material retrieval. 


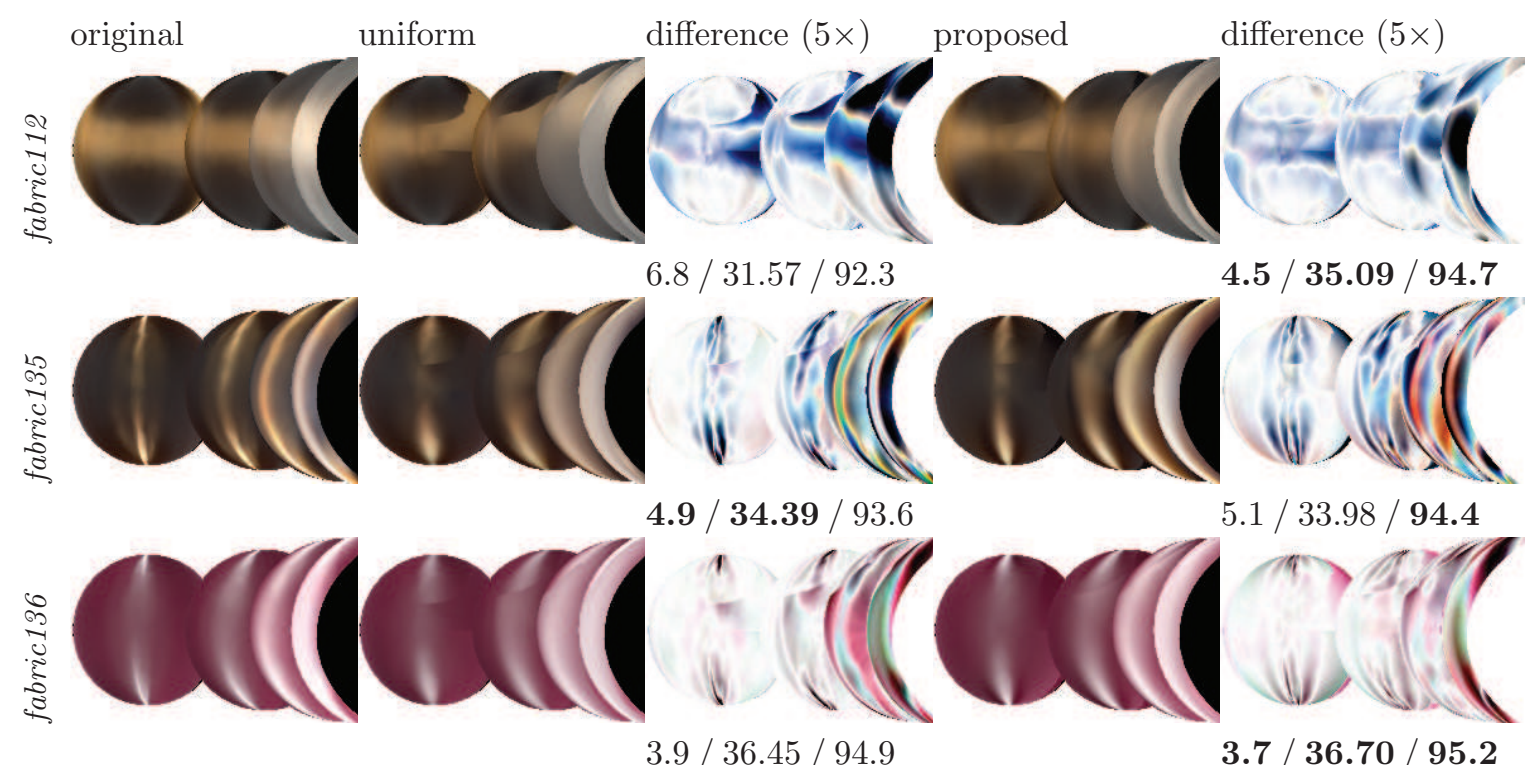

Figure 9. Comparison of renderings of reference BRDF and its reconstructions using 1025 uniform or 1025 anisotropic reciprocal samples, respectively. Below are shown results of RMSE / PSNR [dB] / VDP2 computational metrics.

\section{Acknowledgments}

This research has been supported by the Czech Science Foundation grants 14-02652S and 14-10911S.

\section{REFERENCES}

[1] Nicodemus, F., Richmond, J., Hsia, J., Ginsburg, I., and Limperis, T., "Geometrical considerations and nomenclature for reflectance," NBS Monograph 160, National Bureau of Standards, U.S. Dept. of Com. , 1-52 (1977).

[2] Dana, K., van Ginneken, B., Nayar, S., and Koenderink, J., "Reflectance and texture of real-world surfaces," ACM Transactions on Graphics 18(1), 1-34 (1999).

[3] Filip, J. and Vavra, R., "Template-based sampling of anisotropic BRDFs," Computer Graphics Forum 33, 91-99 (October 2014).

[4] Ward, G., "Measuring and modeling anisotropic reflection," Computer Graphics 26, 265 - 272 (July 1992 ).

[5] Lu, R., Koenderink, J. J., and Kappers, A. M. L., "Optical properties (bidirectional reflectance distribution functions) of velvet.," Applied Optics 37, 5974-5984 (1998).

[6] Lu, R., Koenderink, J. J., and Kappers, A. M., "Specularities on surfaces with tangential hairs or grooves," Computer Vision and Image Understanding 78(3), 320-335 (2000).

[7] Cook, R. and Torrance, K., "A reflectance model for computer graphics," ACM SIGGRAPH 1981, ACM Press 15, 307-316 (August 1981).

[8] Torrance, K. and Sparrow, E., "Theory for off-specular reflection from rough surfaces," Journal of the Optical Society of America 57, 1105-1114 (February 1967).

[9] Kurt, M., Szirmay-Kalos, L., and Křivánek, J., "An anisotropic BRDF model for fitting and monte carlo rendering," ACM Trans. on Comp. Graphics 44, 3:1-3:15 (February 2010).

[10] Raymond, B., Guennebaud, G., Barla, P., Pacanowski, R., and Granier, X., "Optimizing brdf orientations for the manipulation of anisotropic highlights," in [Computer Graphics Forum], 33(2), 313-321, Wiley Online Library (2014).

[11] Ngan, A., Durand, F., and Matusik, W., "Experimental analysis of BRDF models," Eurographics Symposium on Rendering 2005 2, 117-126 (2005).

[12] Filip, J., Vavra, R., and Havlicek, M., "Effective acquisition of dense anisotropic BRDF," in [Proceedings of the 22th International Conference on Pattern Recognition, ICPR 2014], 2047-2052 (August 2014). 
[13] Han, J. Y. and Perlin, K., "Measuring bidirectional texture reflectance with a kaleidoscope," ACM Transactions on Graphics 22(3), 741-748 (2003).

[14] Dana, K., "BRDF/BTF measurement device," in [Proceedings of Eighth IEEE International Conference on Computer Vision. ICCV 2001], 2, 460-466 (July 2001).

[15] Mukaigawa, Y., Sumino, K., and Yagi, Y., "Multiplexed illumination for measuring BRDF using an ellipsoidal mirror and a projector," in [Proceedings of the 8th Asian conference on Computer vision - Volume Part II], ACCV'07, 246-257, Springer-Verlag, Berlin, Heidelberg (2007).

[16] Yasuhiro, M., Sumino, K., and Yasushi, Y., "Rapid BRDF measurement using an ellipsoidal mirror and a projector," IPSJ Trans. on Computer Vision and Appl. 1, 21-32 (2009).

[17] Ghosh, A., Heidrich, W., Achutha, S., and OToole, M., "A basis illumination approach to BRDF measurement," International Journal of Computer Vision 90(2), 183-197 (2010).

[18] Mukaigawa, Y., Tagawa, S., Kim, J., Raskar, R., Matsushita, Y., and Yagi, Y., "Hemispherical confocal imaging using turtleback reflector," in [Computer Vision ACCV 2010], Kimmel, R., Klette, R., and Sugimoto, A., eds., Lecture Notes in Computer Science 6492, 336-349, Springer Berlin Heidelberg (2011).

[19] Mukaigawa, Y., Sumino, K., and Yagi, Y., "High-speed measurement of brdf using an ellipsoidal mirror and a projector," in [Computer Vision and Pattern Recognition, 200\%. CVPR '0\%. IEEE Conference on], 1-8 (June 2007). 